\title{
BMJ Open Diet quality indices, genetic risk and risk of cardiovascular disease and mortality: a longitudinal analysis of 77004 UK Biobank participants
}

\author{
Katherine M Livingstone (D , ${ }^{1}$ Gavin Abbott, ${ }^{1}$ Steven J Bowe, ${ }^{2}$ Joey Ward, ${ }^{3}$ \\ Catherine Milte, ${ }^{1}$ Sarah A McNaughton ${ }^{1}$
}

To cite: Livingstone KM, Abbott G, Bowe SJ, et al. Diet quality indices, genetic risk and risk of cardiovascular disease and mortality: a longitudinal analysis of 77004 UK Biobank participants. BMJ Open 2021;11:e045362. doi:10.1136/ bmjopen-2020-045362

- Prepublication history and additional materials for this paper is available online. To view these files, please visit the journal online (http://dx.doi org/10.1136/bmjopen-2020045362).

Received 29 September 2020 Revised 17 December 2020 Accepted 17 March 2021

Check for updates

(c) Author(s) (or their employer(s)) 2021. Re-use permitted under CC BY-NC. No commercial re-use. See rights and permissions. Published by BMJ.

${ }^{1}$ Institute for Physical Activity and Nutrition, Deakin University, Geelong, Victoria, Australia ${ }^{2}$ Deakin Biostatistics Unit, Deakin University, Geelong, Victoria, Australia

${ }^{3}$ Institute of Health and Wellbeing, University of Glasgow, Glasgow, UK

Correspondence to Dr Katherine M Livingstone; k.livingstone@deakin.edu.au

\section{ABSTRACT}

Objectives To examine associations of three diet quality indices and a polygenic risk score with incidence of allcause mortality, cardiovascular disease (CVD) mortality, myocardial infarction (MI) and stroke.

Design Prospective cohort study.

Setting UK Biobank, UK.

Participants 77004 men and women (40-70 years) recruited between 2006 and 2010.

Main outcome measures A polygenic risk score was created from 300 single nucleotide polymorphisms associated with CVD. Cox proportional HRs were used to estimate independent effects of diet quality and genetic risk on all-cause mortality, CVD mortality, MI and stroke risk. Dietary intake (Oxford WebQ) was used to calculate Recommended Food Score (RFS), Healthy Diet Indicator (HDI) and Mediterranean Diet Score (MDS).

Results New all-cause $(n=2409)$ and CVD $(n=364)$ deaths and $\mathrm{MI}(\mathrm{n}=1141)$ and stroke $(\mathrm{n}=748)$ events were identified during mean follow-ups of 7.9 and 7.8 years, respectively. The adjusted HR associated with one-point higher RFS for all-cause mortality was 0.96 ( $95 \% \mathrm{Cl}: 0.94$ to 0.98 ), CVD mortality was 0.94 (95\% Cl: 0.90 to 0.98$)$, Ml was 0.97 (95\% Cl: 0.95 to 1.00) and stroke was $0.94(95 \%$ Cl: 0.91 to 0.98$)$. The adjusted HR for all-cause mortality associated with one-point higher HDI and MDS was 0.97 ( $95 \%$ Cl: 0.93 to 0.99 ) and 0.95 (95\% Cl: 0.91 to 0.98 ), respectively. The adjusted HR associated with one-point higher MDS for stroke was 0.93 (95\% Cl: 0.87 to 1.00$)$. There was little evidence of associations between $\mathrm{HDI}$ and risk of CVD mortality, MI or stroke. There was evidence of an interaction between diet quality and genetic risk score for Ml.

Conclusion Higher diet quality predicted lower risk of allcause mortality, independent of genetic risk. Higher RFS was also associated with lower risk of CVD mortality and MI. These findings demonstrate the benefit of following a healthy diet, regardless of genetic risk.

\section{INTRODUCTION}

Cardiovascular disease (CVD) is one of the leading causes of morbidity and mortality worldwide. ${ }^{1}$ As a multifactorial condition, CVD risk is attributable to a combination of genetic and behavioural influences. ${ }^{2}$ With
Strengths and limitations of this study

- This large prospective population-based cohort included repeat dietary assessments, using a validated questionnaire and hospital register data on cardiovascular disease (CVD) incidence and mortality.

- The creation of three contrasting diet quality indices informs the best practice design and implementation of food-based diet quality indices for assessing diet-disease relationships.

- A polygenic genetic risk score was created for each participant using 300 single-nucleotide polymorphisms known to be associated with CVD and allcause mortality.

- The present analysis is likely to be subject to selfselection bias associated with the number of participants who completed the dietary assessment and the low response rate.

- Findings are not generalisable to non-Caucasian populations, thus, future research in diverse populations is needed to investigate the applicability of different diet quality methodologies for examining CVD risk independent of genetic susceptibility.

poor diet now a leading risk factor for noncommunicable diseases, ${ }^{3}$ further understanding of the role of diet on CVD risk is warranted.

The overall quality of diets is an emerging predictor of CVD events and mortality. ${ }^{45}$ Diet quality indices, that score dietary intakes according to a priori knowledge, ${ }^{6}$ have been used to investigate association between diet and CVD incidence and mortality. ${ }^{45-11}$ These indices can capture different aspects of diet quality, for example, being based on intakes for encouraged foods only (eg, Recommended Food Score, RFS), a combination of foods and nutrients from dietary guidelines (eg, Healthy Diet Indicator, HDI) or a dietary pattern identified as healthful (eg, Mediterranean Diet Score, MDS). However, our 
understanding is limited by the use of contrasting diet quality methodologies and a paucity of studies comparing different indices in large prospective population-based cohorts. Comparison of contrasting diet quality indices will identify whether differences in these methodologies are important for understanding diet-disease associations and will inform the international standardisation of diet quality methodologies for assessing health outcomes. ${ }^{5712}$

The role of diet and genetics on risk of CVD is an emerging area of research. ${ }^{13}{ }^{14}$ Prior to the accessibility to whole genome sequencing, most research focused on links between single nucleotide polymorphisms (SNPs) and CVD. ${ }^{15-17}$ Recent research has shown that polygenic risk scores (PRS), that incorporate multiple SNPs, are a good indicator of risk for complex conditions, such as CVD,${ }^{14} 18$ although the extent to which they influence the association between diet quality and CVD risk is unclear. Further research is also needed to elucidate whether diet quality is a risk factor for CVD independent of genetic risk. Moreover, the longitudinal association between contrasting diet quality indices, genetic risk and different CVD subtypes is unknown. Thus, the aim of this study was to examine the prospective role of three Diet Quality Indices (HDI, RFS and MDS) and a PRS on risk of stroke, myocardial infarction (MI), CVD mortality and all-cause mortality. Findings will advance understanding of the applicability of diet quality indices for assessing CVD risk.

\section{METHODS}

\section{Study design and participants}

The UK Biobank is a population cohort of half a million individuals living in the UK that aimed to examine determinants of disease in middle-aged adults. ${ }^{19}$ Persons aged 40-69 years were identified from National Health Service patient registers and invited to participate. Individuals were invited to 1 of 22 assessment centres across England, Scotland and Wales between 2006 and 2011. At each centre, participants completed a touchscreen questionnaire to collect information on demographic characteristics, lifestyle behaviours and general health. The Oxford WebQ a web-based 24 hours dietary assessment tool, was introduced in 2009 to collect information on dietary intake. ${ }^{20}$ Physical measurements (eg, height and weight) were taken and participants provided blood and urine samples. Participants were followed up via linkage to health records and death registries. Electronic signed consent was obtained from all participants. Participants were excluded from the present analysis if they (1) did not identify as White British, (2) were ineligible based on previous history of CVD before entering the study, pregnancy, implausible physical activity data and CVD events during the study prior to completion of last dietary questionnaire, (3) had missing data for outcomes, exposures and covariates/moderators and (4) had less than two timepoints of dietary data between February 2011 and June 2012. Results are reported according to the Strengthening the Reporting of Observational Studies in Epidemiology-Nutritional Epidemiology checklist for cohort studies. ${ }^{21}$

\section{Study measures}

\section{Dietary intake}

The Oxford WebQ was used to collect information on the frequency of consumption of 206 foods and 32 beverages during the previous 24 hours. ${ }^{2022} 23$ The Oxford WebQ is a 24-hour dietary questionnaire that has been validated against a traditional interviewer-administered multiplepass 24-hour dietary recall and biomarkers for protein, potassium and total sugar intake and total energy expenditure estimated by accelerometery. ${ }^{23}$ Energy and nutrient intakes were calculated by multiplying the frequency of consumption of each food or drink by the standard portion size and energy and nutrient composition of each item. ${ }^{24}{ }^{25}$ Participants recruited between April 2009 and September 2010 completed the Oxford WedQ using the touchscreen at the assessment centre. Repeat Oxford WebQs were collected via four online cycles between February 2011 and June 2012: February 2011 and April 2011 (online cycle 1); June 2011 and September 2011 (online cycle 2); October 2011 and December 2011 (online cycle 3); April 2012 and June 2012 (online cycle $4)$. The total period of available dietary data from the Oxford WebQ was 38 months (April 2009-June 2012). Email invitations were sent on different days of the week to capture variation in dietary intakes and participants were given 3 days to complete the questionnaire for cycles 1 and 2 and 14 days for cycles 3 and 4 .

To establish a baseline dietary intake in the present analysis, we calculated a mean dietary intake based on the four online Oxford WebQ cycles only. This was because the time between the first and fourth online cycle measurements was 16 months (February 2011-Jun 2012) and was considered a more credible timeframe for an average baseline than the 38 months available from all five Oxford WebQ measurements. This resulted in a minimal sample loss $(<10 \%)$ while providing a shorter dietary exposure period and a more consistent approach to the use of the dietary data by using only the online cycles of the OxfordWebQ. To better capture usual intake, we calculated average nutrient intakes, food group intakes and diet quality scores for participants who had two or more valid measurements for the four online cycles of the Oxford WebQ.

\section{Diet quality}

Information on food and beverage intakes from the Oxford WebQ were used to calculate three diet quality indices: the RFS, which is based on intakes of encouraged foods only, ${ }^{26}$ and the HDI, which scores intakes of a combination of foods and nutrients from dietary guidelines, ${ }^{27}$ and the MDS, representing dietary patterns identified as healthful. ${ }^{28}$ These indices were selected as they represent three contrasting diet quality methodologies that have been applied internationally to assess diet-disease associations. ${ }^{9} 1026272930$ 
The RFS is a food-based variety index designed to assess consumption of food groups encouraged in the dietary guidelines. ${ }^{10}$ As detailed in online supplemental table 1 , food intakes were scored according to five food groups: fruits (seven items), vegetables (seven items), wholegrains (two items), lean meat and alternatives (three items) and reduced fat dairy products (two items). Scoring was based on the RFS designed by Kant and Graubard ${ }^{26}$ and has been used elsewhere. ${ }^{31}{ }^{32}$ We summed intakes of food items within each group to create a total intake for each food group. Food groups were then assigned a score of 1 if they were consumed above the minimum amount threshold: $15 \mathrm{~g} /$ day for non-beverages and $30 \mathrm{~g} /$ day for beverages. Intakes below these thresholds were scored 0 . Scores ranged between 0 and 21, with higher scores indicating a higher quality diet and a wider consumption of recommended foods. ${ }^{33}$

The HDI is a food-based and nutrient-based index designed to reflect consumption of foods recommended for a healthy diet by WHO. ${ }^{34}$ The original HDI was developed and validated in 1997 based on the 1990 WHO's dietary recommendations for the prevention of chronic disease. ${ }^{35}$ We adapted a 12-point Healthy Diet Score designed by Maynard $e t a l^{27}$ to reflect adherence to the 2020 WHO healthy diet fact sheet. ${ }^{34}$ As cholesterol intake is not part of the 2020 recommendations and information on its intake was not available in the UK Biobank, we used an 11-item score that included the following groups: saturated fat; polyunsaturated fat; protein; total carbohydrates; dietary fibre; fruits and vegetables; pulses and nuts; total non-milk extrinsic sugars; fish; red meat and meat products; and calcium. Data on intake of non-milk extrinsic sugars were not available in the UK Biobank and so we adapted the HDI to score intakes of total sugars instead. Criteria for scoring was based on cut points detailed in online supplemental table 2. We assigned intakes within the cut offs a score of 1 and those outside of the cut offs were assigned a score of 0 . The total score ranged from 0 to 11 , with a higher score reflecting a higher diet quality (online supplemental table 2).

The MDS is a food-based and nutrient-based score designed to reflect adherence to a Mediterranean style diet. The present study used the 9-item index developed and validated by Trichopoulou $e t a l$ as it is the first and most widely used version of the MDS. ${ }^{36}{ }^{37}$ Food and nutrient intakes were scored according to nine components: vegetables, legumes, fruits and nuts, cereals, fish and seafood, monounsaturated fats to saturated fats ratio, dairy products, meat and meat products and alcohol (online supplemental table 3). As used by Trichopoulou $e t a l^{36}$ we used sex-specific median intakes as cut-off points for intakes of each component. A score of 1 was assigned to participants whose intake of vegetables, legumes, fruits and nuts, cereals, fish and seafood and monounsaturated: saturated fats was above the median. A score of 1 was assigned to intake of dairy products, meat and meat products below the median. For alcohol, a score of 1 was assigned for low to moderate intake (intake of no more than two times/day). A score of 0 was assigned for no alcohol intake or intake greater than two times per day. ${ }^{38}$ Total MDS score ranged from 0 to 9 , with higher scores reflecting better alignment to the Mediterranean diet.

\section{Cardiovascular events and mortality}

Mortality status and causes of death were determined by linkage of data with the UK National Death Index (NDI) using the most recent available data from the UK Biobank (September 2020). The accuracy of the NDI for identifying CVD deaths has been established previously in Australia. ${ }^{39}$ CVD mortality was estimated from 2006 International Classification of Diseases 10th revision (ICD-10) codes in death certificates. CVD mortality was identified using ICD codes I05-I89. CVD events were recorded between enrolment (1999-2000) and the most recent inpatient hospital data available from the UK Biobank (September 2020). Incident MI (ST-Elevation MI and Non-ST-Elevation MI) and stroke (ischaemic, intracerebral haemorrhage and subarachnoid haemorrhage) were available from algorithms provided by the UK Biobank. ${ }^{40}{ }^{41}$ Algorithms were produced to reliably identify incidence of selected illnesses through consideration of hospital and death register data. The adjudication of 'algorithmically defined' outcomes for MI and stroke are detailed elsewhere. ${ }^{40} 41$ A censoring data of 4 March 2020 was used for all outcomes. This date was chosen due to a spike in deaths from 5 March onwards, which is likely to correspond to increasing deaths due to COVID-19 recorded in the $\mathrm{UK}^{42}$

\section{Polygenic risk score}

We used the March 2018 release of the imputed genetic data from UK Biobank (downloaded 11 November 2019). From the resulting dataset, we excluded those who selfreported ancestry other than white British, those who were missing more than $10 \%$ of the genetic data and those who were defined by UK Biobank as being heterozygosity outliers. Additionally, for every pair of who were individuals who were second cousins or closer (ie, those with a kinship coefficient $>0.042$ ) one was excluded at random. We used information on 300 SNPs known to be associated with coronary artery disease ${ }^{43}$ to create a PRS for CVD for each individual. ${ }^{44}$ Evidence indicates that a genetic risk score estimated from these 300 SNPs is associated with traditional risk factors for CVD, such as type 2 diabetes and hypertension, contributes to the development of CVD conditions that have their origins in atherosclerosis, such as peripheral arterial disease and stroke, and is associated with premature mortality. ${ }^{43}$ The PRS was estimated using PLINK, an open-source tool for genomic research, ${ }^{45}$ by generating the sum of the number of risk alleles present at each locus and weighting by the log of the odds for that locus ${ }^{18}$ estimated from the list of 300 SNPs using the PLINK '-score' command-with no-mean-imputation flag. PRS were available for all participants included in the final study sample, where PRS were transformed to 
standardised $\mathrm{Z}$ scores and were treated as a continuous variable in all modelling.

\section{Demographic and health information}

Information on demographics, medical history and health behaviours were collected using interview-administered questionnaires at recruitment and follow ups. Participant age at recruitment and sex were self-reported. No adjustments were made for discrepancies between selfreported sex and genetic sex. Education was assessed by asking 'Which of the following qualifications do you have? (You can select more than one),' with the options college or university degree, A levels or equivalent, $\mathrm{O}$ levels or GCSEs or equivalent, CSEs, National Vocational Qualification (NVQ)/Higher National Diploma (HND) / Higher National Certificate (HNC), other professional qualifications (eg, nursing or teaching). We operationalised this into five categories based on the highest level of education: (1) college or university degree, (2) all professional qualification $(\mathrm{NVQ} / \mathrm{HND} / \mathrm{HNC}$, other professional qualifications), (3) A levels or equivalent, (4) O levels, GCSEs or equivalent or CSEs and (5) none of the above or prefer not to answer. The Townsend deprivation index, a composite measure of deprivation based on unemployment, non-car ownership, non-home ownership and household overcrowding, ${ }^{46}$ was estimated from the preceding national census data, with each participant assigned a score corresponding to the postcode of their home dwelling and a negative value representing high socioeconomic status. We operationalised the Townsend deprivation index as quintiles.

Information on smoking (never, previous and current smoker), previous doctor diagnosis of any type of diabetes or a CVD event (yes, no) and use of medication (antihypertensive, lipid-lowering or exogenous hormones or diabetes; yes, no) were collected. We created a binary variable for family history (of father, mother and siblings) of CVD and related diseases (yes, no). Body mass index (BMI) was calculated as weight $/$ height $^{2}$ collected during the Assessment Centre visit. We created a binary variable to indicate overweight or obese according to standard WHO cut offs. ${ }^{47}$ Physical activity was estimated using metabolic equivalents (METs), the ratio of a person's working metabolic rate relative to their resting metabolic rate. One MET was defined as the energy cost of sitting quietly and is equivalent to a caloric consumption of 1 $\mathrm{kcal} / \mathrm{kg} /$ hour. We used standard cut-offs to categorise participants as meeting physical activity guidelines of 150 min per week if their METs were $\geq 600$ MET-min/week. ${ }^{48}$

\section{Statistical analysis}

Complete case analysis was used. We investigated missingness by comparing demographic characteristics of the excluded sample with the analytic sample. Descriptive analyses included mean (SD) for continuous variables and number (\%) for categorical variables. We created sample-based tertiles of diet quality for RFS, HDI and MDS for descriptive purposes only. Unadjusted linear regression analyses were used to examine intakes of encouraged food groups and total energy and nutrient intakes across tertiles of diet quality indices. This descriptive analysis aimed to show that diet quality scores reflect differences in underlying food and nutrient intakes, thus assisting with interpretation and translation into actual food intakes.

We used multivariable Cox proportional hazard regression models to estimate HRs and 95\% CIs of all-cause mortality, CVD mortality and risk of CVD events (MI and stroke) according to each diet quality index separately (RFS, HDI and MDS). We treated diet quality indices as continuous independent variables. CVD events and mortality were treated as time-to-event outcome/dependent variables. We estimated the duration of follow-up as the time between the last day of dietary data and the first event of either an MI, stroke, mortality or the censoring date (4 March 2020). In participants who had multiple events during the study period, the first event date was used. We adjusted the Cox regression analyses for covariates identified using a directed acyclic graph (online supplemental figure 1). These included age (continuous), sex, deprivation (categorical), smoking status (categorical), physical activity (continuous), medication use (binary), family history of CVD (binary) and energy intake (continuous). The role of sex by diet quality and by PRS interactions were further tested by adding an interaction term to each model. Consistent with recommendations for sex differences in cardiovascular associations, ${ }^{49}$ analyses were presented stratified by sex regardless of whether there were any apparent sex differences. The Cox proportional hazards models also included PRS as an independent variable and were additionally adjusted for the first eight principal components of ancestry and genotyping batch. ${ }^{14}$ We included an interaction term in the models to test for statistical interaction between each diet quality score and PRS. Interactions were further inspected by conducting post-hoc estimation of the effects of diet quality indices on events at 'low' and 'high' PRS score of -1 and +1 which, given PRS was a standard score, represent minus and plus one SD of PRS. Data were analysed using Stata (V.16.0; StataCorp). To address possible reverse causation, sensitivity analyses excluded deaths and incident cases of MI and stroke within the first 2 years of follow-up.

\section{Patient and public involvement}

The development of the research question or outcome measures was not informed by patients' priorities, experience or preferences. No patients were involved in the design and conduct of the present study. There are no plans to disseminate the results to study participants.

\section{RESULTS}

Of the 502536 participants who were recruited into the UK Biobank, 425529 participants were excluded based on being not white British ( $\mathrm{n}=92$ 907), having 
Table 1 Baseline characteristics of participants in the UK Biobank

\begin{tabular}{|c|c|c|c|}
\hline Characteristic & $\begin{array}{l}\text { Overall } \\
\text { N (\%) }\end{array}$ & $\begin{array}{l}\text { Males } \\
\text { N (\%) }\end{array}$ & $\begin{array}{l}\text { Females } \\
\mathbf{N}(\%)\end{array}$ \\
\hline$n$ & 77004 & $34984(45.4)$ & $42020(54.5)$ \\
\hline \multicolumn{4}{|l|}{ Highest level of education } \\
\hline College or university degree & $36709(47.8)$ & $17271(49.4)$ & $19438(46.3)$ \\
\hline Professional qualifications & $7467(9.7)$ & $4062(11.6)$ & $3405(8.1)$ \\
\hline None/prefer not to answer & $4454(5.8)$ & $2082(6.0)$ & $2372(5.7)$ \\
\hline \multicolumn{4}{|l|}{ Townsend Deprivation Index } \\
\hline Least deprived & $18129(23.5)$ & $8611(24.6)$ & $9518(22.7)$ \\
\hline Most deprived & $10681(13.9)$ & $4756(13.6)$ & $5926(14.1)$ \\
\hline \multicolumn{4}{|l|}{ Smoking } \\
\hline Never smoked & $44856(58.3)$ & 18849 (53.9) & 26007 (61.9) \\
\hline Ex-smoker & $27184(35.3)$ & $13471(38.5)$ & $13714(32.6)$ \\
\hline Current smoker & $4964(6.5)$ & $2664(7.6)$ & $2300(5.5)$ \\
\hline Body mass index $\left(\mathrm{kg} / \mathrm{m}^{2}\right)$, mean $\pm \mathrm{SD}^{*}$ & $26.5 \pm 4.4$ & $27.1 \pm 3.9$ & $26.0 \pm 4.7$ \\
\hline Waist circumference $(\mathrm{cm})$, mean $\pm \mathrm{SD}^{\star}$ & $88.1 \pm 13.0$ & $95.2 \pm 10.8$ & $82.3 \pm 11.6$ \\
\hline Total physical activity (MET min), mean \pm SD & $2477 \pm 2326$ & $2542 \pm 2439$ & $2423 \pm 2227$ \\
\hline
\end{tabular}

A levels/AS levels, advanced levels/AS levels; O levels/GCSE/CSEs, ordinary levels/GCES/general certificate of education; professional qualifications include national vocational qualification/higher national diploma/higher national certificate, other professional qualifications; Townsend Deprivation Index is a composite measure of deprivation based on unemployment, non-car ownership, non-homeownership and household overcrowding.

${ }^{*}$ Data on body mass index and waist circumference were available in $n=76901$ and $n=76950$ respectively. †Medication use was restricted to lipid lowering or blood pressure.

AS, advanced subsidiary; CVD, cardiovascular disease; GCSE, General Certificate of Secondary Education; METs, metabolic equivalents.

unusable genetic data $(\mathrm{n}=1459)$, being ineligible $(\mathrm{n}=23$ 215) or missing dietary or covariate data $(n=307951$; online supplemental figure 2). Excluded participants were similar in age and sex to the included sample, with somewhat higher BMI and rates of smoking and deprivation (online supplemental table 4). A total of 77 004 participants were included in the present analysis (table 1). Mean age at recruitment was 56.2 (SD 7.8) years and $55 \%$ were female. Forty-eight per cent of participants had a colleague or university degree, most were experiencing low to mid deprivation $(67 \%)$, had never smoked $(58 \%)$ and had a family history of CVD (74\%; table 1). Fifty-nine per cent of the participants were overweight or obese and $85 \%$ met physical activity guidelines.

Mean RFS, HDI and MDS were 6.78 (SD 2.40), 3.57 (SD 1.26) and 5.31 (SD 1.04), respectively. Intake of fruits, vegetables, wholegrains and lean meat were higher with increasing tertile of RFS, HDI and MDS (table 2). Intake of low-fat dairy were higher with increasing tertile of RFS and HDI and lower with increasing tertile of MDS. Intakes of total fat, saturated fat, carbohydrates and sugars were higher with increasing tertile of RFS, HDI and MDS. Intakes of protein were lower with increasing tertile of HDI and MDS, while intakes were higher with increasing tertile of RFS. Intakes of polyunsaturated fat were lower with increasing tertile of RFS, while intakes were higher with increasing tertile of HDI and MDS (table 2).

During a mean follow-up of 7.8 years (a total of 600 193 person-years), we observed 1141 new MI events and 748 new stroke events. During a mean follow-up of 7.9 years (a total of 604431 person-years), we observed 364 deaths due to CVD and 2409 all-cause deaths. Of these, the majority of MI $(72 \%)$ and stroke $(60 \%)$ events, and CVD $(72 \%)$ and all-cause $(59 \%)$ deaths were in males. 


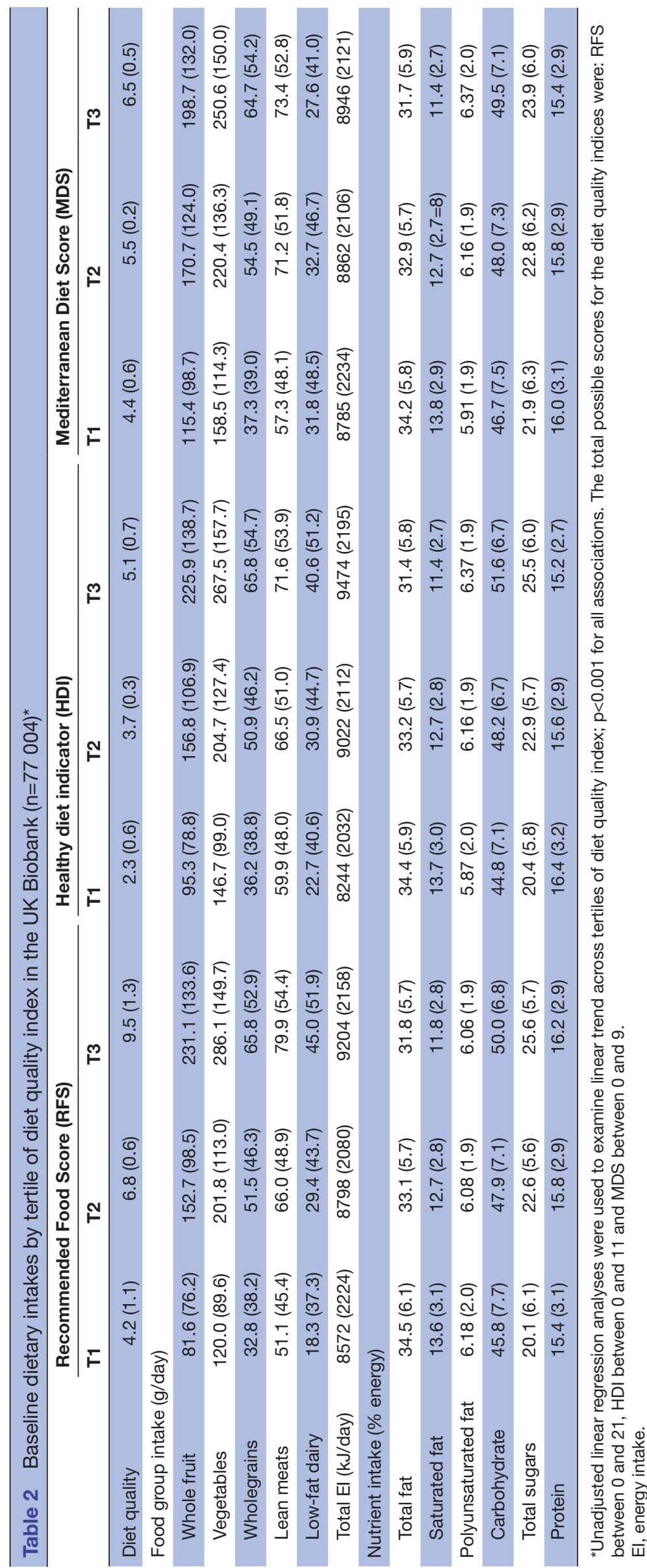


Table 3 Cox-proportional HRs and 95\% Cl for risk of all-cause mortality, CVD mortality and CVD events with increasing Recommended Food Score (RFS) and Polygenic Risk Score in participants from the UK Biobank



Models were adjusted for age (continuous), sex (when not used to stratify), deprivation (categorical), smoking status (categorical), physical activity (continuous), medication use (binary), family history of CVD (binary), energy intake (continuous) and the first eight principal components of ancestry and genotyping batch. All models include main effects of diet quality and polygenic risk score but not interaction terms.

CVD, cardiovascular disease.

\section{RFS and risk of all-cause mortality, CVD mortality, MI and stroke}

The adjusted HR associated with a one-point higher RFS for all-cause mortality was 0.96 (95\% CI: 0.94 to 0.98 ), for CVD mortality was 0.94 (95\% CI: 0.90 to 0.98 ), for MI was 0.97 (95\% CI: 0.95 to 1.00 ) and for stroke was 0.94 (95\% CI: 0.91 to 0.98 ) (table 3). There was limited evidence (all $\mathrm{p}>0.1$ ) of sex by diet interactions. When stratified by sex, associations were comparable in men, while there was only evidence of an association between RFS and all-cause mortality and stroke in females. The adjusted HR associated with a one-point higher PRS for MI was 1.33 (95\% CI: 1.25 to 1.41); when stratified by sex, there was evidence of a stronger association in males. When an interaction term for PRS was added to the models, there was no evidence (at the $\mathrm{p}<0.05$ level) of interaction between RFS and PRS for any outcomes ( $p$ interaction $=0.40$ (all-cause mortality), $\mathrm{p}$ interaction $=0.77$ (CVD mortality), $\mathrm{p}$ interaction $=0.17$ (MI) and $\mathrm{p}$ interaction $=0.10$ (stroke) $)$. The interaction of sex by PRS showed evidence that the effect of higher PRS on higher risk of MI was more pronounced for males $(\mathrm{HR}=1.21,95 \% \mathrm{CI}$ : 1.06 to $1.37, \mathrm{p}=0.004)$. Effect sizes were consistent when deaths and incident cases of MI and stroke within the first 2 years of follow-up were excluded (data not shown).

\section{HDI and risk of all-cause mortality, CVD mortality, MI and stroke}

The adjusted HR associated with a one-point higher HDI for all-cause mortality was 0.97 (95\% CI: 0.93 to 0.99 ). There was little evidence of associations between HDI and risk of CVD mortality, MI or stroke (table 4). There was limited evidence (all $\mathrm{p}>0.1$ ) of sex by diet interactions. When stratified by sex, there was evidence of an association between HDI and all-cause mortality in males only. The adjusted HR associated with a one-point higher PRS for MI was 1.33 (95\% CI: 1.25 to 1.41 ), which when stratified by sex, there was evidence of a stronger association in males. When an interaction term for PRS was added to the models, there was no evidence of interaction between HDI and PRS for other outcomes ( $p$ interaction $=0.66$ (allcause mortality), $\mathrm{p}$ interaction $=0.86$ (CVD mortality) and $\mathrm{p}$ interaction $=0.17$ (stroke) $)$. There was some evidence of interaction between HDI and PRS for MI events (p-interaction $=0.049$ ). While there was no evidence of an effect of HDI on MI for participants with low ( -1 SD) PRS (HR=1.02, 95\% CI: 0.95 to $1.10, p=0.61$ ), there was some evidence of an association between higher HDI and reduced risk of MI events for those with high (+1 SD) PRS $(\mathrm{HR}=0.93,95 \%$ CI: 0.88 to $0.99, \mathrm{p}=0.017)$. The interaction of sex by PRS showed evidence that the effect of higher PRS on higher risk of MI was more pronounced for males 
Table 4 Cox proportional HRs and $95 \% \mathrm{Cl}$ for risk of all-cause mortality, CVD mortality and CVD events with increasing Healthy Diet Indicator (HDI) and Polygenic Risk Score in participants from the UK Biobank

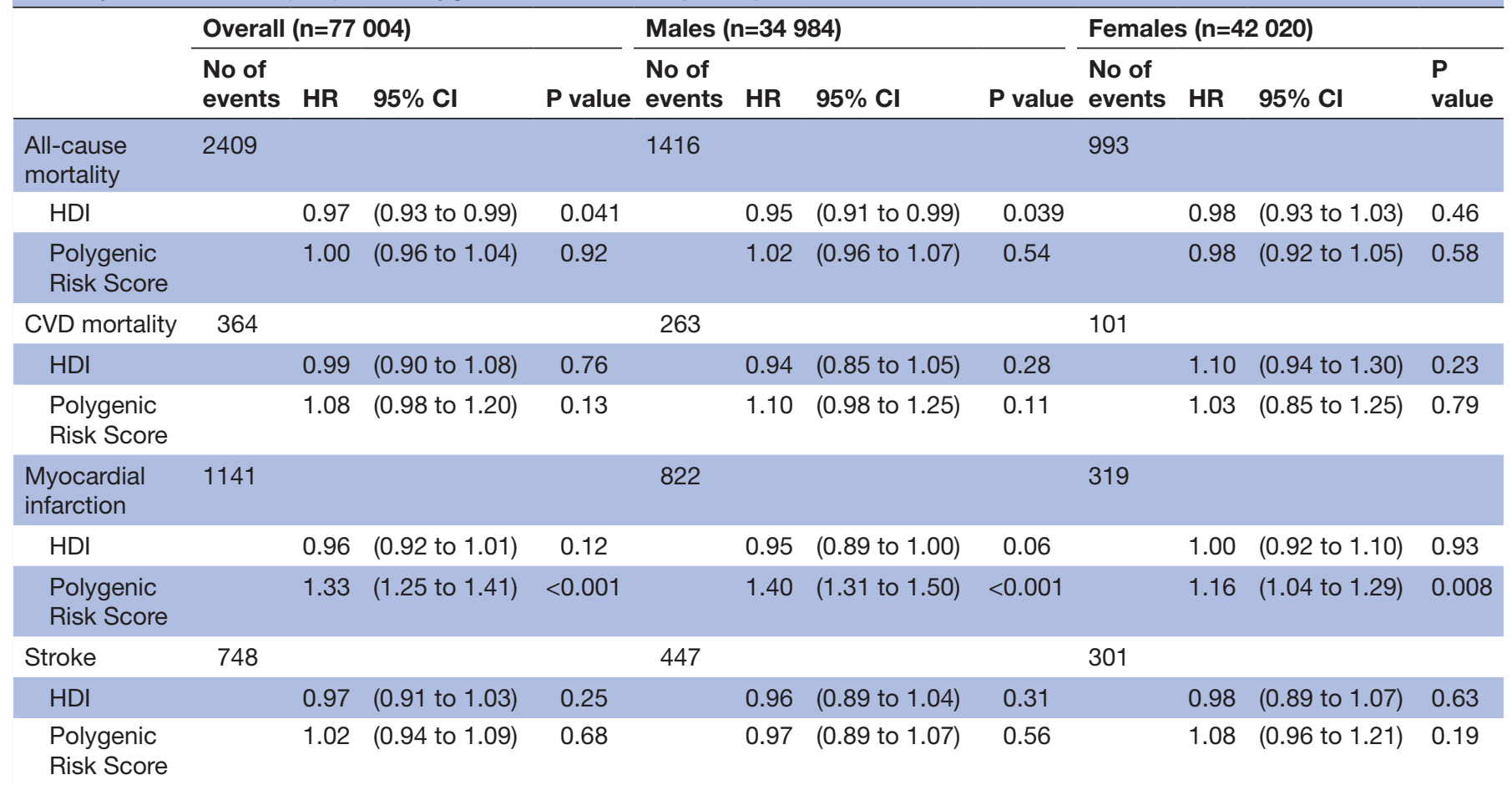

Models were adjusted for age (continuous), sex (when not used to stratify), deprivation (categorical), smoking status (categorical), physical activity (continuous), medication use (binary), family history of CVD (binary), energy intake (continuous) and the first eight principal components of ancestry and genotyping batch. All models include main effects of diet quality and polygenic risk score but not interaction terms.

CVD, cardiovascular disease.

$(\mathrm{HR}=1.21,95 \% \mathrm{CI}: 1.06$ to $1.37, \mathrm{p}=0.004)$. Effect sizes were consistent when deaths and incident cases of MI and stroke within the first 2 years of follow-up were excluded (data not shown).

\section{MDS and risk of all-cause mortality, CVD mortality, MI and stroke}

The adjusted HR associated with a one-point higher MDS for all-cause mortality was 0.95 (95\% CI: 0.91 to 0.98$)$ and for stroke was 0.93 (95\% CI: 0.87 to 1.00 ) (table 5 ). There was limited evidence of associations between MDS and risk of CVD mortality and MI. There was limited evidence (all $\mathrm{p}>0.1$ ) of sex by diet interactions. When stratified by sex, there was evidence of an association between MDS and all-cause mortality and MI in males only. The adjusted HR associated with a one-point higher PRS for MI was 1.33 (95\% CI: 1.25 to 1.41$)$; when stratified by sex, there was evidence of a stronger association in males. When an interaction term for PRS was added to the models, there was no evidence of interaction between MDS and PRS for other outcomes ( $p$ interaction=0.58 (all-cause mortality), $\mathrm{p}$ interaction $=0.72$ (CVD mortality) and $\mathrm{p}$ interaction $=0.12$ (stroke) $)$. There was evidence of interaction between MDS and PRS for MI events ( $p$-interaction=0.026). While there was no evidence of an effect of MDS on MI for those with low ( $-1 \mathrm{SD}) \mathrm{PRS}(\mathrm{HR}=1.03$, 95\% CI: 0.94 to $1.12, \mathrm{p}=0.56)$ there was strong evidence of an association between higher MDS and reduced risk of MI events for those with high $(+1 \mathrm{SD}) \mathrm{PRS}(\mathrm{HR}=0.91$, 95\% CI: 0.85 to $0.97, \mathrm{p}=0.004)$. The interaction of sex by PRS showed evidence that the effect of higher PRS on higher risk of MI was more pronounced for males $(\mathrm{HR}=1.21,95 \% \mathrm{CI}: 1.06$ to $1.37, \mathrm{p}=0.004)$. Effect sizes were consistent when deaths and incident cases of MI and stroke within the first 2 years of follow-up were excluded (data not shown).

\section{DISCUSSION}

This prospective population-based cohort study aimed to examine the association of three diet quality indices (RFS, HDI and MDS) and genetic risk score with incidence of CVD and mortality. Our main findings were that higher RFS, HDI and MDS were associated with lower risk of mortality, regardless of genetic CVD risk. However, only the RFS showed evidence of lower risk of CVD mortality, MI and stroke, suggesting the applicability of the diet quality indices may depend on the health outcome in question. We also identified that increasing genetic risk of CVD was associated with MI only. There was some evidence suggesting that the underlying genetics of both MI and death may follow different pathways in males and females. Interaction analyses suggested that following a 


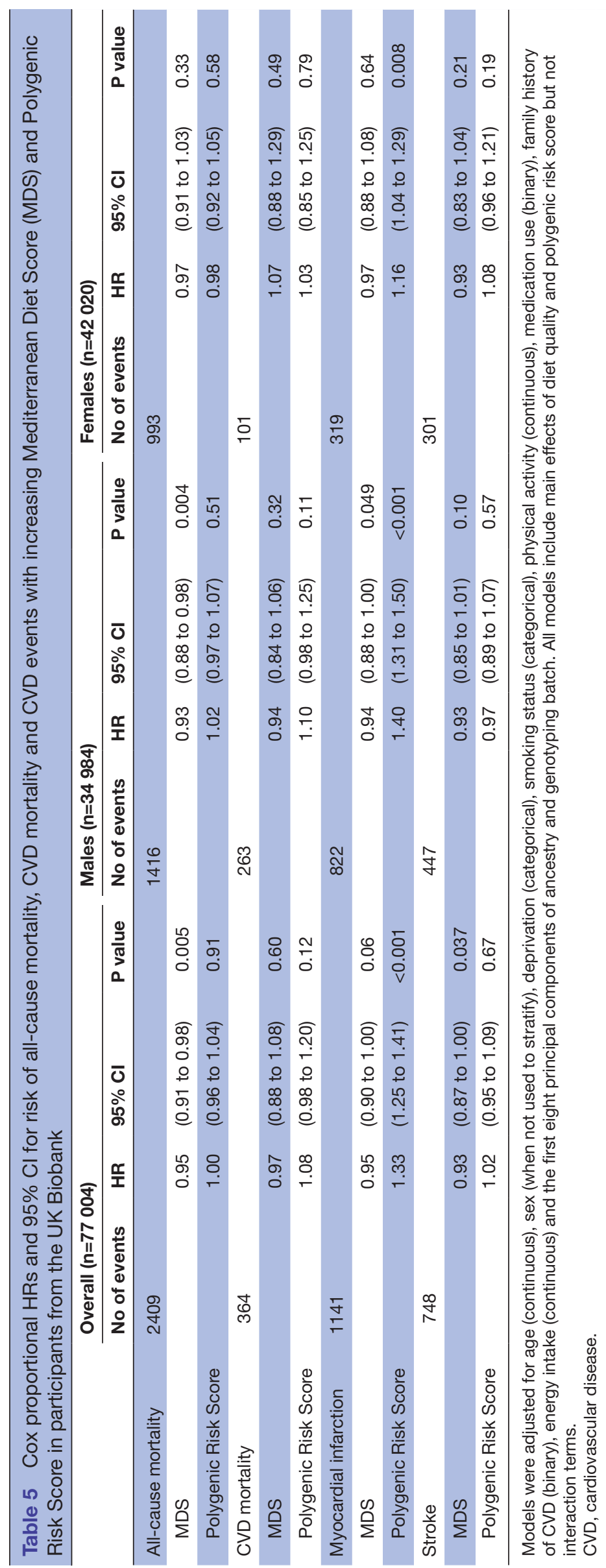


healthy diet may be of particular importance for reducing risk of MI in individuals with high genetic risk of CVD. Nonetheless, our findings demonstrate the benefit of following a healthy diet independent of genetic risk.

Our findings for reduced risk of all-cause mortality with higher diet quality are consistent with previous research on the MDS, ${ }^{595051} \mathrm{HDI}^{5052}$ and RFS. ${ }^{53}$ Moreover, a comparison of 10 diet quality indices in over 450 000 European adults showed that all indices examined were inversely associated with 10-year risk of all-cause mortality. ${ }^{52}$ In the present study, the predictive role of diet quality on risk of all-cause mortality remained after adjusting for major non-modifiable determinants of allcause mortality, including age, sex and family history of CVD. This highlights the importance of modifiable risk factors for death, regardless of whether the diet quality index is based on intakes of encouraged foods (ie, RFS), foods and nutrients from dietary guidelines (ie, HDI) or a dietary pattern identified as healthful (ie, MDS). Moreover, the common elements across all three indices is the inclusion of food-based components, such as fruit and vegetables and lean meat and alternatives, rather than nutrients, affirming the value of food-based dietary guidelines in preventative healthcare rather a reductionist nutrient-based approach. ${ }^{54}$

Evidence for an association between diet quality indices and CVD risk is mixed. ${ }^{5711143255-57}$ Confirming our findings, large-scale studies in the UK population have shown independent associations between healthy diets and lifestyles and low genetic risk in reducing risk of CVD, with mixed results for interactions. ${ }^{1457}$ Only one study to date has used an overall diet quality index, ${ }^{11}$ with comparable results to the present study, highlighting the potential to include plant-based diet quality components when assessing diet-disease associations. ${ }^{56} 5859$ Future diet-disease research should extend this to better understand the role of specific plant and animal foods as part of overall dietary patterns. Moreover, the UK Biobank participants are predominately white and highly educated and genetic risk profiles and their associations with risk of CVD may be different in more diverse populations. Thus, further research in diverse populations is needed to investigate the applicability of these diet quality methodologies for examining CVD risk independent of genetic risk. ${ }^{7}$

Although there was limited evidence of interactions for sex by diet quality index, our stratified results showed large effect sizes for associations between diet quality and genetic CVD risk in males. This warrants further investigation as previous research shows stronger associations in males than females. ${ }^{1460}$ The role of sex may be partly explained by the high prevalence of diabetes and unhealthy behaviours in men, ${ }^{61}$ and in this study may be due the lower number of events and deaths in women compared with men. Nonetheless, it is possible that the biological and behavioural pathways in which risk factors exert their effects on CVD risk are different between men and women. ${ }^{60}$

\section{Strengths and limitations}

Our main strength was the large sample size and inclusion of genetic data. This enabled investigation of a genetic risk score created using 300 SNPs known to be associated with CVD, more than any previous publications in the UK Biobank. ${ }^{1457}$ While the PRS used was specific to coronary disease, it has been used to identify predispositions to a wide variety of CVD and non-CVDs, as well as premature mortality, given these may develop in parallel with coronary disease for the same genetic origins. The dietary questionnaire has been previously validated and included sufficient detail to allow us to create three contrasting diet quality indices. There are a number of limitations that should be acknowledged. While the dietary assessment method is a short-term measure of intake, our use of up to four instances of dietary assessments provided an estimate of longer-term intake. Although the present analysis is likely to be subject to self-selection bias associated with the number of participants who completed the dietary assessment and the low response rate, associations between demographic and behavioural risk factors and mortality in the UK Biobank have been shown to be comparable to those from national health survey data from England and Scotland. ${ }^{62}$ While we adjusted analyses based on a range of confounders identified using a directed acyclic graph, we cannot discount the possibility of residual or unmeasured confounding.

\section{Conclusion}

This prospective population-based cohort study provided evidence that higher diet quality (RFS, HDI and MDS) was associated with lower risk of all-cause mortality, regardless of genetic CVD risk. Diet quality, when estimated using the RFS only, was associated with lower risk of CVD mortality and MI, independent of genetic CVD risk. The diet quality indices investigated in this study have common food-based scoring components, providing further evidence for the best practice design and implementation of food-based diet quality indices for assessing health outcomes. Further research in diverse populations is needed to investigate the applicability of different diet quality methodologies for examining CVD risk independent of genetic susceptibility.

\section{Twitter Katherine M Livingstone @katmlivingstone}

Contributors KML, GA, SJB, JW, CM and SAM designed the analysis. KML, SJB, JW and GA conducted the statistical analysis. KML drafted the manuscript. All authors contributed to the critical review of the manuscript and approved the final version of the manuscript.

Funding KL is supported by a National Health and Medical Research Council Emerging Leadership Fellowship (APP1173803). JW is funded by the Lister Prize Fellowship (173096).

Disclaimer The funding source had no role in the design or conduct of the study; collection, management, analysis, and interpretation of the data; or preparation, review, or approval of the manuscript.

Competing interests All authors have completed the ICMJE uniform disclosure form at www.icmje.org/coi_disclosure.pdf and declare: no support from any organisation for the submitted work; KML has been a consultant for HeadUp Labs; no other financial relationships with any organisations that might have an interest 
in the submitted work in the previous 3 years; no other relationships or activities that could appear to have influenced the submitted work.

\section{Patient consent for publication Not required}

Ethics approval UK Biobank received ethical approval from the research ethics committee (reference 13/NW/0382). An ethics exemption was granted by Deakin University Human Research Ethics Committee (Reference number 2019_293).

Provenance and peer review Not commissioned; externally peer reviewed.

Data availability statement Data may be obtained from a third party and are not publicly available. The genetic and phenotypic UK Biobank data are available on application to the UK Biobank. This research has been conducted using the UK Biobank Resource under Application 34894

Supplemental material This content has been supplied by the author(s). It has not been vetted by BMJ Publishing Group Limited (BMJ) and may not have been peer-reviewed. Any opinions or recommendations discussed are solely those of the author(s) and are not endorsed by BMJ. BMJ disclaims all liability and responsibility arising from any reliance placed on the content. Where the content includes any translated material, BMJ does not warrant the accuracy and reliability of the translations (including but not limited to local regulations, clinical guidelines, terminology, drug names and drug dosages), and is not responsible for any error and/or omissions arising from translation and adaptation or otherwise.

Open access This is an open access article distributed in accordance with the Creative Commons Attribution Non Commercial (CC BY-NC 4.0) license, which permits others to distribute, remix, adapt, build upon this work non-commercially, and license their derivative works on different terms, provided the original work is properly cited, appropriate credit is given, any changes made indicated, and the use is non-commercial. See: http://creativecommons.org/licenses/by-nc/4.0/.

\section{ORCID iD}

Katherine M Livingstone http://orcid.org/0000-0002-9682-7541

\section{REFERENCES}

1 Roth GA, Abate D, Abate KH. Global, regional, and national agesex-specific mortality for 282 causes of death in 195 countries and territories, 1980-2017: a systematic analysis for the global burden of disease study 2017. Lancet 2018;392:1736-88.

2 Joseph P, Leong D, McKee M. Reducing the global burden of cardiovascular disease, part 1: the epidemiology and risk factors. Circ Res 2017;121:677-94.

3 Afshin A, Sur PJ, Fay KA. Health effects of dietary risks in 195 countries, 1990-2017: a systematic analysis for the global burden of disease study 2017. Lancet 2019;393:1958-72.

4 Sotos-Prieto M, Bhupathiraju SN, Mattei J, et al. Changes in diet quality scores and risk of cardiovascular disease among US men and women. Circulation 2015;132:2212-9.

5 Miller V, Webb P, Micha R. Defining diet quality: a synthesis of dietary quality metrics and their validity for the double burden of malnutrition. Lancet Planetary Health 2020;4:e352-70.

6 Tapsell LC, Neale EP, Satija A, et al. Foods, nutrients, and dietary patterns: interconnections and implications for dietary guidelines. Adv Nutr 2016;7:445-54.

7 Aljuraiban GS, Gibson R, Oude Griep LM. Perspective: the application of a priori diet quality scores to cardiovascular disease Risk - A critical evaluation of current scoring systems. Adv Nutr 2019;11:10-24.

8 Aigner A, Becher $\mathrm{H}$, Jacobs S. Low diet quality and the risk of stroke mortality: the multiethnic cohort study. Eur J Clin Nutr 2018;72:1035-45.

9 Sotos-Prieto M, Bhupathiraju SN, Mattei J. Association of changes in diet quality with total and cause-specific mortality. $N$ Engl J Med 2017;377:143-53.

10 Kant AK, Schatzkin A, Graubard BI. A prospective study of diet quality and mortality in women. JAMA 2000;283:2109-15.

11 Heianza Y, Zhou T, Sun D. Genetic susceptibility, plant-based dietary patterns, and risk of cardiovascular disease. Am J Clin Nutr 2020;112:220-8.

12 Liese AD, Krebs-Smith SM, Subar AF, et al. The dietary patterns methods project: synthesis of findings across cohorts and relevance to dietary guidance. J Nutr 2015;145:393-402.

13 Voruganti VS. Nutritional genomics of cardiovascular disease. Curr Genet Med Rep 2018:6:98-106.

14 Rutten-Jacobs LC, Larsson SC, Malik R, et al. Genetic risk, incident stroke, and the benefits of adhering to a healthy lifestyle: cohort study of 306473 UK Biobank participants. BMJ 2018;363:k4168.
15 Ahmad T, Chasman DI, Mora S, et al. The fat-mass and obesityassociated (FTO) gene, physical activity, and risk of incident cardiovascular events in white women. Am Heart J 2010;160:1163-9.

16 Song Y, Stampfer MJ, Liu S. Meta-Analysis: apolipoprotein E genotypes and risk for coronary heart disease. Ann Intern Med 2004;141:137-47.

17 Cambien François, Tiret L. Genetics of cardiovascular diseases. Circulation 2007;116:1714-24.

18 Sugrue LP, Desikan RS. What are polygenic scores and why are they important? JAMA 2019;321:1820-1.

19 Sudlow C, Gallacher J, Allen N. UK Biobank: an open access resource for identifying the causes of a wide range of complex diseases of middle and old age. PLoS Med 2015;12:e1001779.

20 Bradbury KE, Young HJ, Guo W, et al. Dietary assessment in UK Biobank: an evaluation of the performance of the touchscreen dietary questionnaire. J Nutr Sci 2018;7:e6.

21 Lachat C, Hawwash D, Ocké MC. Strengthening the reporting of observational studies in Epidemiology - Nutritional epidemiology (STROBE-nut): an extension of the STROBE statement. PLoS Med 2016;13:e1002036.

22 Liu B, Young H, Crowe FL. Development and evaluation of the Oxford WebQ, a low-cost, web-based method for assessment of previous $24 \mathrm{H}$ dietary intakes in large-scale prospective studies. Public Health Nutr 2011;14:1998-2005.

23 Greenwood DC, Hardie LJ, Frost GS, et al. Validation of the Oxford WebQ online 24-hour dietary questionnaire using biomarkers. Am $J$ Epidemiol 2019;188:1858-67.

24 Food Standards Agency. Food portion sizes. London: The Stationary Office, 2005

25 Royal Society of Chemistry; Ministry of Agriculture FaFCambridge. McCance and widdowson's the composition of foods. 6th edn. Roya Society of Chemistry, 2002.

26 Kant AK, Graubard BI. A comparison of three dietary pattern indexes for predicting biomarkers of diet and disease. J Am Coll Nutr 2005;24:294-303.

27 Maynard M, Gunnell D, Ness AR. What influences diet in early old age? Prospective and cross-sectional analyses of the Boyd Orr cohort. Eur J Public Health 2005;16:315-23.

28 Panagiotakos DB, Pitsavos C, Arvaniti F, et al. Adherence to the Mediterranean food pattern predicts the prevalence of hypertension, hypercholesterolemia, diabetes and obesity, among healthy adults; the accuracy of the MedDietScore. Prev Med 2007;44:335-40.

29 Waijers PMCM, Feskens EJM, Ocké MC. A critical review of predefined diet quality scores. Br J Nutr 2007;97:219-31.

30 Kanauchi M, Kanauchi K. The World Health Organization's Healthy Diet Indicator and its associated factors: A cross-sectional study in central Kinki, Japan. Prev Med Rep 2018;12:198-202.

31 McNaughton SA, Bates CJ, Mishra GD. Diet quality is associated with all-cause mortality in adults aged 65 years and older. $J$ Nutr 2012;142:320-5

32 Livingstone KM, McNaughton SA. Diet quality is associated with obesity and hypertension in Australian adults: a cross sectional study. BMC Public Health 2016;16:1037.

33 McCullough ML, Feskanich D, Stampfer MJ. Diet quality and major chronic disease risk in men and women: moving toward improved dietary guidance. Am J Clin Nutr 2002;76:1261-71.

34 World Health Organization. Healthy diet WHO fact sheet. Available: http://www.who.int/mediacentre/factsheets/fs394/en/ [Accessed 9 Mar 2020].

35 Huijbregts P, Feskens E, Räsänen L, et al. Dietary pattern and 20 year mortality in elderly men in Finland, Italy, and the Netherlands: longitudinal cohort study. BMJ 1997;315:13-17.

36 Trichopoulou A, Orfanos P, Norat T, et al. Modified Mediterranean diet and survival: EPIC-elderly prospective cohort study. BMJ 2005;330:991.

37 Trichopoulou A, Costacou T, Bamia C. Adherence to a Mediterranean diet and survival in a Greek population 2003;348:2599-608.

38 Bach-Faig A, Berry EM, Lairon D, et al. Mediterranean diet pyramid today. science and cultural updates. Public Health Nutr 2011;14:2274-84.

39 Magliano D, Liew D, Pater H, et al. Accuracy of the Australian National death index: comparison with adjudicated fatal outcomes among Australian participants in the long-term intervention with pravastatin in ischaemic disease (LIPID) study. Aust N Z J Public Health 2003;27:649-53.

40 Schnier C, Bush K, Nolan J. Definitions of stroke for UK Biobank phase 1 outcomes adjudication documentation prepared by: on behalf of UK Biobank outcome adjudication group, 2017. Available: http://biobank.ndph.ox.ac.uk/showcase/showcase/docs/alg outcome_mi.pdf [Accessed 16 Sep 2020]. 
41 Schnier C, Bush K, Nolan J. Definitions of acute myocardial infarction and main myocardial infarction pathological types UK Biobank phase 1 outcomes adjudication documentation prepared by: on behalf of UK Biobank outcome adjudication group, 2017. Available: http:// biobank.ndph.ox.ac.uk/showcase/showcase/docs/alg_outcome_mi. pdf [Accessed 16 Sep 2020].

42 National Health Service. COVID-19 daily deaths, 2020. Available: https://www.england.nhs.uk/statistics/statistical-work-areas/covid19-daily-deaths/ [Accessed 16 Sep 2020].

43 Ntalla I, Kanoni S, Zeng L. Genetic risk score for coronary disease identifies predispositions to cardiovascular and Noncardiovascular diseases. J Am Coll Cardiol 2019;73:2932-42.

44 Dudbridge F. Polygenic epidemiology 2016;40:268-72.

45 Purcell S, Neale B, Todd-Brown K. PLINK: a tool set for wholegenome association and population-based linkage analyses. Am J Hum Genet 2007;81:559-75.

46 Towsend P, Phillimore P, Beattie A. Health and deprivation. Nurs Stand 1988;2:34

47 World Health Organization. BMI classification 2015. Available: http:// apps.who.int/bmi/index.jsp?introPage=intro_3.html [Accessed 3 Dec 2015].

48 The IPAQ Group. IPAQ scoring protocol - International physical activity questionnaire. Available: https://sites.google.com/site/ theipaq/scoring-protocol [Accessed 22 May 2020].

49 Woodward M. Rationale and tutorial for analysing and reporting sex differences in cardiovascular associations. Heart 2019;105:1701-8.

50 Neelakantan N, Koh W-P, Yuan J-M, et al. Diet-quality indexes are associated with a lower risk of cardiovascular, respiratory, and allcause mortality among Chinese adults. J Nutr 2018;148:1323-32.

51 Stefler D, Malyutina S, Kubinova R. Mediterranean diet score and total and cardiovascular mortality in Eastern Europe: the HAPIEE study. Eur J Nutr 2017;56:421-9.
52 Lassale C, Gunter MJ, Romaguera D. Diet quality scores and prediction of all-cause, cardiovascular and cancer mortality in a panEuropean cohort study. PLoS One2016;11:e0159025.

53 Kaluza J, Håkansson N, Brzozowska A. Diet quality and mortality: a population-based prospective study of men. Eur J Clin Nutr 2009;63:451-7.

54 Schulze MB, Martínez-González MA, Fung TT, et al. Food based dietary patterns and chronic disease prevention. BMJ 2018;361:k2396.

55 Lakkur S, Judd SE. Diet and stroke 2015;46:2007-11.

56 Chiavaroli L, Viguiliouk E, Nishi SK. Dash dietary pattern and cardiometabolic outcomes: an umbrella review of systematic reviews and meta-analyses. Nutrients 2019;11

57 Said MA, Verweij N, van der Harst P. Associations of combined genetic and lifestyle risks with incident cardiovascular disease and diabetes in the UK Biobank study. JAMA Cardiol 2018;3:693-702.

58 Baden MY, Liu G, Satija A. Changes in plant-based diet quality and total and cause-specific mortality. Circulation 2019;140:979-91.

59 Huang T, Yang B, Zheng J. Cardiovascular disease mortality and cancer incidence in vegetarians: a meta-analysis and systematic review. Ann Nutr Metab 2012;60:233-40.

60 Den Ruijter HM, Haitjema S, Asselbergs FW. Sex matters to the heart: a special issue dedicated to the impact of sex related differences of cardiovascular diseases. Atherosclerosis 2015;241:205-7.

61 Andersen KK, Andersen ZJ, Olsen TS, et al. And gender-specific prevalence of cardiovascular risk factors in 40102 patients with firstever ischemic stroke. Stroke 2010;41:2768-74.

62 Batty GD, Gale CR, Kivimäki M, et al. Comparison of risk factor associations in UK Biobank against representative, general population based studies with conventional response rates: prospective cohort study and individual participant meta-analysis. BMJ 2020;368:m131. 